\title{
COMPOSITION OF CORONAL MASS EJECTIONS
}

\author{
T. H. Zurbuchen ${ }^{1}$, M. Weberg ${ }^{1}$, R. von Steiger $^{2,3}$, R. A. Mewaldt ${ }^{4}$, S. T. Lepri ${ }^{1}$, and S. K. Antiochos \\ ${ }^{1}$ Department of Climate and Space Sciences and Engineering, University of Michigan, Ann Arbor, MI, USA \\ ${ }_{2}^{2}$ International Space Science Institute, Bern, Switzerland \\ ${ }_{4}^{3}$ Physikalisches Institut, University of Bern, Switzerland \\ ${ }^{4}$ California Institute of Technology, Pasadena, CA, USA \\ ${ }^{5}$ Heliophysics Science Division, NASA Goddard Space Flight Center, Greenbelt, MD, USA \\ Received 2016 January 18; revised 2016 April 27; accepted 2016 May 4; published 2016 July 14
}

\begin{abstract}
We analyze the physical origin of plasmas that are ejected from the solar corona. To address this issue, we perform a comprehensive analysis of the elemental composition of interplanetary coronal mass ejections (ICMEs) using recently released elemental composition data for $\mathrm{Fe}, \mathrm{Mg}, \mathrm{Si}, \mathrm{S}, \mathrm{C}, \mathrm{N}, \mathrm{Ne}$, and $\mathrm{He}$ as compared to $\mathrm{O}$ and $\mathrm{H}$. We find that ICMEs exhibit a systematic abundance increase of elements with first ionization potential (FIP) $<10 \mathrm{eV}$, as well as a significant increase of Ne as compared to quasi-stationary solar wind. ICME plasmas have a stronger FIP effect than slow wind, which indicates either that an FIP process is active during the ICME ejection or that a different type of solar plasma is injected into ICMEs. The observed FIP fractionation is largest during times when the Fe ionic charge states are elevated above $Q_{\mathrm{Fe}}>12.0$. For ICMEs with elevated charge states, the FIP effect is enhanced by $70 \%$ over that of the slow wind. We argue that the compositionally hot parts of ICMEs are active region loops that do not normally have access to the heliosphere through the processes that give rise to solar wind. We also discuss the implications of this result for solar energetic particles accelerated during solar eruptions and for the origin of the slow wind itself.
\end{abstract}

Key words: acceleration of particles - solar wind - Sun: abundances - Sun: coronal mass ejections (CMEs)

\section{INTRODUCTION}

Coronal mass ejections (CMEs) constitute the largest explosive releases of mass and energy in the solar system. During a time period of tens of minutes, CMEs of masses up to $10^{12} \mathrm{~kg}$ can be accelerated to speeds of $2000 \mathrm{~km} \mathrm{~s}^{-1}$ or more. The physical process through which magnetic energy is transferred into the kinetic energy of these ejecta is still debated, but it most likely involves reconnection in the lower corona, which can lead to significant heating of coronal plasmas, as observed in UV and X-ray radiation and shown in Figure 1. The observations imply plasma temperatures well above the coronal temperatures of $1 \mathrm{MK}$. The CME plasma can also undergo rapid acceleration, as indicated in coronagraph observations, also shown in Figure 1. Various parts of CMEs are visible, including ejected neutrals in filaments, which have large densities. The accelerating CME plasma interacts with the plasma populations in the corona, often leading to shock waves, which are thought to accelerate particles to relativistic energies.

Shock-associated gradual solar energetic particle (SEP) events have important space weather effects on spacecraft and humans in space. Particles accelerated in conjunction with CMEs tend to have the composition of the coronal and heliospheric plasma from which they are accelerated (Reames 1994), but they can also include some particles that are much more representative of so-called impulsive events, which are associated with flares (Cohen et al. 1999). On average, gradual energetic particles show strong fractionation relative to photospheric composition, with particles of first ionization potential (FIP) $<10 \mathrm{eV}$ (also referred to as low-FIP elements) enhanced by approximately a factor of 2.7-3.5 relative to the photosphere. In these measurements, elements with FIP larger than $10 \mathrm{eV}$ are depleted relative to $\mathrm{O}$ at approximately $80 \%$ and $\mathrm{He}$ at $50 \%$ compared to the photospheric composition. Similarly, $\mathrm{Ne}$ is reduced relative to $\mathrm{O}$ as compared to what is believed to be the photospheric abundance, as derived from in situ samples (Shearer et al. 2014).

A similar FIP-dependent compositional pattern is observed also in streamer-associated slow wind (Geiss et al. 1995; Von Steiger et al. 2000), but with a significantly lower magnitude. Low-FIP elements are enhanced by a factor of 1.5-2.5 over photospheric values, not by a factor of 4 , and $\mathrm{Ne}$ and $\mathrm{He}$ are again depleted relative to expected photospheric values. Von Steiger et al. (2000) also pointed out that, in contrast, solar wind originating from polar coronal holes exhibits much lower fractionation, with a FIP effect of 1-1.5 for low-FIP elements. However, $\mathrm{Ne}$ and $\mathrm{He}$ were again observed to be depleted to values comparable to those of slow, streamer-associated wind.

An important recent result on photospheric composition and FIP enhancements and their comparisons with in situ compositions makes historic comparisons rather difficult. Due to the development of photospheric spectral inversion techniques using 3D fluid models, and also taking into account nonthermal effects, the photospheric composition derived from spectroscopic data has changed substantially over time (Asplund 2005; Von Steiger \& Zurbuchen 2016). For example, O/H is reduced by up to $40 \%$. These abundances pose some important challenges, as they are no longer consistent with metallicity constraints from helioseismology. Additionally, a recent analysis of in situ $\mathrm{Ne}$ concluded that these newly published abundances pose significant challenges to explain in situ $\mathrm{Ne}$ measurements (Shearer et al. 2014). For these reasons, and for continuity with previous work, we will therefore use the same photospheric abundances used previously by Von Steiger et al. (2000), which are from Grevesse \& Sauval (1998). When comparing with composition from coronal holes and streamers, we use observations recently published by Von Steiger \& Zurbuchen (2016). It is straightforward to adjust our data to any 


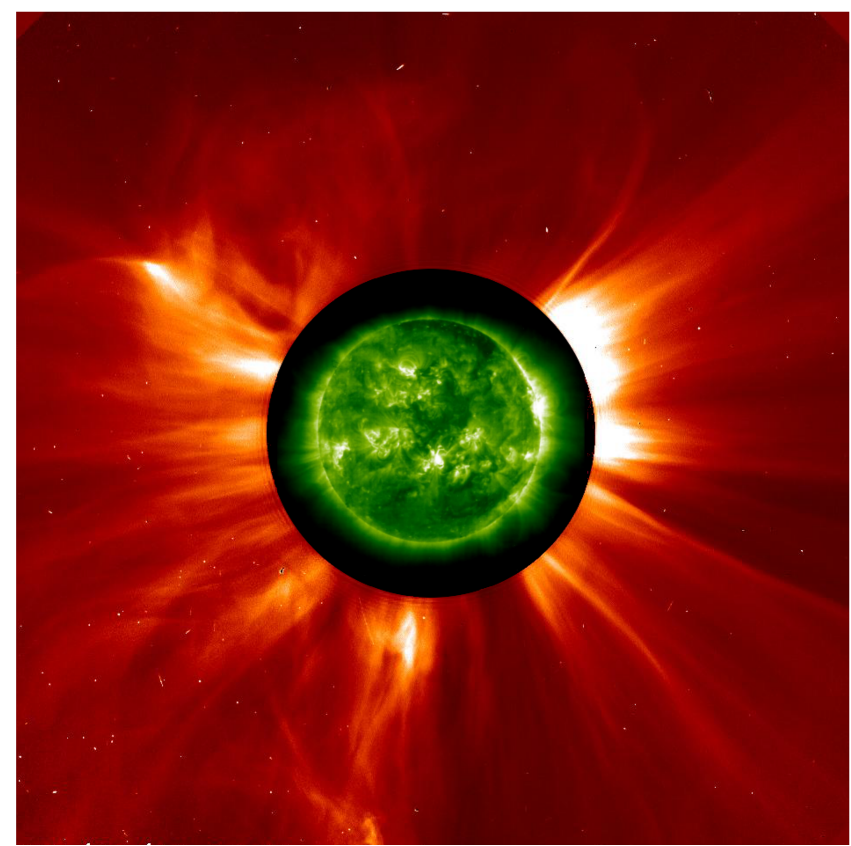

Figure 1. Coronal observations of a coronal mass ejection observed using EIT $195 \mathrm{~A}$ at $15: 36$ UT on day 99.65 of year 2001. The outer image was taken by LASCO C2 at 18:29 UT on day 100.27. This halo CME from S21W4 is associated with an ICME observed approximately two days later at 19:00 UT, after an average transit speed of $1290 \mathrm{~km} \mathrm{~s}^{-1}$.

photospheric data set once the confidence in the new composition is sufficiently high.

Interplanetary coronal mass ejections (ICMEs) are the heliospheric counterparts to CMEs observed near the Sun. These ICMEs are often indicative of magnetically dominated or even cloud-like ejecta that expand into the heliosphere and interact with the ambient solar wind. The ejecta tend to have lower plasma temperatures than the ambient solar wind, indicative of overexpansion, and they have highly elevated ionic charge states, indicative of strong heating near the Sun (Zurbuchen \& Richardson 2006 and references therein). Of particular interest is the enhancement of ionic charge states of $\mathrm{O}$, with increases in both $\mathrm{O}^{7+}$ and $\mathrm{O}^{8+}$ over the He-like charge state $\mathrm{O}^{6+}$ (Henke et al. 2001), and also those of Fe, with large enhancements of the Ne-like charge state $\mathrm{Fe}^{16+}$ or above. Such elevated ionic charge states increase the average Fe charge state, $\left\langle Q_{\mathrm{Fe}}\right\rangle$, and are virtually absent from solar wind away from ICMEs (Lepri et al. 2001). The association of these signatures with active regions has been shown using both geometric mapping (Reinard 2005) and quantitative analysis of the heating and expansion of CME plasma. In fact, Gruesbeck et al. (2011) showed that in situ compositional measurements are consistent with a temperature history of ICME plasma that includes rapid heating and high density followed by an expansion and cooling into the heliosphere. Gruesbeck et al. (2012) later showed that plasma found with relatively low ionic charge states is not derived from overcooling, but is instead associated with a cold source, such as filaments on the Sun, as discussed in the context of Figure 1.

The elemental composition of ICMEs is of interest due to its diagnostic value for the analysis of the origin and evolution of ICME-associated plasmas, which are released from the magnetically closed part of the corona. Plasma from the closed corona escapes in at least two different ways. On the one hand, it has been shown that there is a quasi-stationary release of such plasma, presumably through interchange reconnection (Zurbuchen 2007 and references therein). According to a recent model for this reconnection process, the so-called S-web model (Antiochos et al. 2011), reconnection occurs along a dynamic interface region about the open-closed boundary of the coronal magnetic field. This region maps out to the heliospheric current sheet and to a complex set of singular arcs in the heliosphere that form the S-web. In these models, active region loops that are low-lying and near a polarity inversion line typically do not undergo interchange reconnection, because the open-closed boundary rarely passes through the interiors of active regions, and never crosses a polarity inversion line (e.g., Antiochos et al. 2007). This is in contrast to the eruption from CMEs, especially fast CMEs, which transiently eject into the heliosphere coronal plasma from filament channels along polarity inversion lines deep inside an active region. Since the magnetic fields surrounding active regions are also involved in the eruption of ICMEs, both active region plasma and the overlying coronal field can be ejected into the heliosphere.

One might expect, therefore, that there are compositional signatures in at least a subset of ICMEs that cannot be found in streamer-associated slow wind. Indeed, there have been some reports of changes in elemental composition in conjunction with ICMEs. Hirshberg et al. (1972) and Borrini et al. (1982) reported intermittent enhancements of $\mathrm{He}^{2+} / \mathrm{H}^{+}>8 \%$ during ICME periods. Ipavich et al. (1986) first reported on $\mathrm{Fe} / \mathrm{O}$ enhancements in 1986, a result that was expanded to multiple ions by Richardson \& Cane (2004). In their analysis both $\mathrm{Ne} / \mathrm{O}$ and $\mathrm{Mg} / \mathrm{O}$ were reportedly increased during ICMEs as compared to solar wind. The analysis also mentioned an overall correlation of $\mathrm{Mg} / \mathrm{O}$ and $\mathrm{He}^{2+} / \mathrm{H}^{+}$enhancements with $\mathrm{O}^{7+} / \mathrm{O}^{6+}$ (see also Zurbuchen et al. 2004).

In this paper we analyze the composition of ICME plasma and compare it to streamer-associated slow wind, as well as fast wind from coronal holes. We use recently released data, which, for the first time, correctly include the inversion of lowabundance species such as $\mathrm{Ne}$, as well as $\mathrm{S}$ and $\mathrm{Si}$, and thus constitute the first in situ data set that has both the temporal resolution and statistical accuracy to analyze the elemental composition of ICME plasma quantitatively. This will be clarified in Section 2. Second, we will discuss the physical implications of the results for the genesis and expansion of ICME plasma in Section 3 and also relate our compositional measurements to those of SEPs.

\section{IN SITU MEASUREMENTS OF ICME PLASMA}

The Solar Wind Ion Composition Spectrometer (SWICS) on board the Advanced Composition Explorer (ACE) has provided the most complete composition data of the in-ecliptic solar wind since its launch and initial checkout period in 1997 (Gloeckler et al. 1998). Using a combination of three measurement techniques - an electrostatic analysis, time-offlight analysis with post-acceleration, and total energy measurement - the instrument is able to measure independently particle speed, mass, and charge for incident particles. Due to the use of triple coincidence rates, background rates are small, even in the presence of large high-energy particle fluxes (Gilbert et al. 2014), allowing a high dynamic range during most conditions and thus providing access to elemental abundances of heavy solar wind species, even during ICME periods. The interpretation of the SWICS data relies strongly 


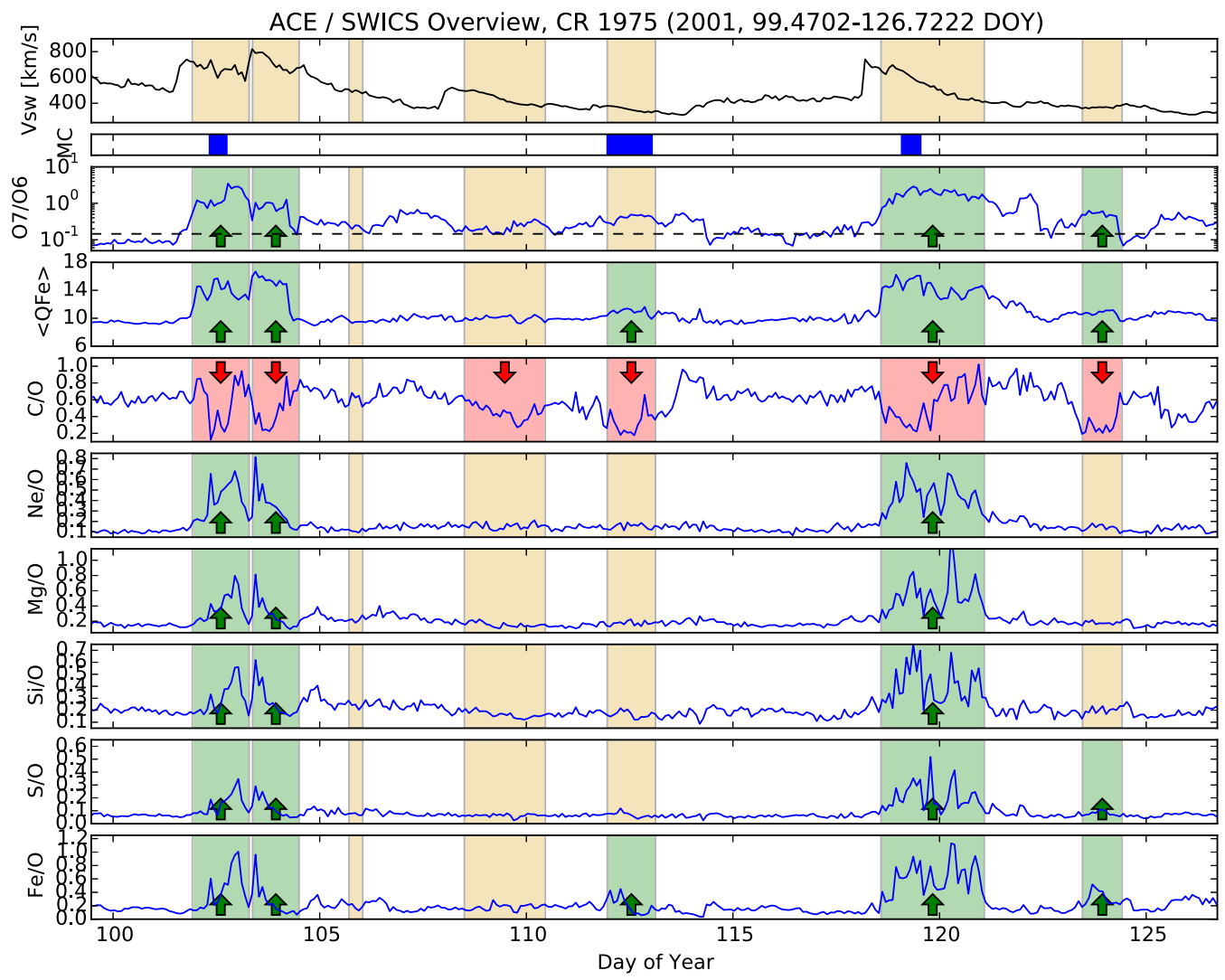

Figure 2. Solar wind plasma and composition measurements for a Carrington rotation in 2001. We use solar wind speed from SWICS; brown shaded time periods are indicative of ICME periods by Richardson and Cane. The blue shaded bars indicate the presence of magnetic cloud signatures identified in the same list. Ionic charge states of $\mathrm{O}$ and $\mathrm{Fe}$ are shown next, followed by the elemental compositional ratios of $\mathrm{C} / \mathrm{O}, \mathrm{Ne} / \mathrm{O}, \mathrm{Mg} / \mathrm{O}, \mathrm{Si} / \mathrm{O}, \mathrm{S} / \mathrm{O}$, and Fe/O. Significant increases and decreases of the elemental composition ratios are marked in green and red, respectively.

on a recently completed statistically accurate inversion model (Shearer et al. 2014), which takes into account both statistical and systematic uncertainties of the instrument. Such an advanced inversion model is particularly important for elements like $\mathrm{S}, \mathrm{N}$, and $\mathrm{Ne}$, though the model is limited by counting statistics and, due to the resolution limits of SWICS, also involves the deconvolution of ion peaks that overlap with much more abundant ions such as $\mathrm{C}$ and $\mathrm{O}$. Figure 2 summarizes plasma, composition, and magnetic field data for the duration of one Carrington rotation starting shortly before day 100 in 2001, near the same time in the solar cycle depicted in Figure 1. During this time period, sunspots and active regions were observed in a wide range of latitudes, and CMEs occurred on a regular basis.

For geometric reasons, only a fraction of these CMEs sweep over $A C E$ and are observed as ICMEs. The in situ signatures of ICMEs are marked in Figure 2 by shaded bars using the list of Richardson \& Cane (2010). This time period includes the CME shown in Figure 1, where it was observed during the time period of DOY 101.9 to $103.3,2001$. Blue bars further indicate ICMEs that have magnetic cloud signatures. Figure 2 also shows solar wind speed, as measured by SWICS, and the ionic charge states of $\mathrm{O}$ (i.e., $\mathrm{O}^{7+} / \mathrm{O}^{6+}$ ) and $\mathrm{Fe}$ (i.e., $\left\langle Q_{\mathrm{Fe}}\right\rangle$ ), which have already been discussed. Green (red) shading indicates ICMEs that show enhancements (depletions) in a given compositional quantity by more than one standard deviation above (below) the average value within non-ICME solar wind inside the same Carrington rotation. As previously discussed, a fraction of ICMEs show statistically significant elevated charge states. The next six panels show the elemental abundances of $\mathrm{C} / \mathrm{O}, \mathrm{Ne} / \mathrm{O}, \mathrm{Mg} / \mathrm{O}, \mathrm{Si} / \mathrm{O}, \mathrm{S} / \mathrm{O}$, and $\mathrm{Fe} / \mathrm{O}$, calculated by summing over all ionic charge states of each element. Again, enhancements and depletions are marked with green and red shadings, respectively. There are two ICMEs, one near DOY 103 and another near 120, that exhibit fractionation in nearly every single compositional parameter. The plasmas exhibit elevated ionic charge states, and all low-FIP elements are increased, as is $\mathrm{Ne} / \mathrm{O}$. Yet there is an apparent depletion of $\mathrm{C} /$ $\mathrm{O}$ suggestive of the effect recently discussed by Zhao et al. (2016). In their analysis, it was shown that there are time periods during which the fully ionized charge states, such as $\mathrm{C}^{6+}$ or $\mathrm{O}^{8+}$, are reduced relative to the other ionic charge states, even though the overall freeze-in temperatures would imply a higher flux of ions that are fully ionized. That reduction most strongly affects $\mathrm{C}^{6+}$, and thus can reduce the overall $\mathrm{C} / \mathrm{O}$ ratio. A detailed analysis of this particular effect is beyond the scope of this investigation, but is the subject of a future publication.

In summary, Figure 2 reveals that there are compositional anomalies within ICMEs well beyond the signatures summarized by Richardson \& Cane (2004) or Zurbuchen \& Richardson (2006). The signatures tend to be most prevalent where elevated ionic charge states are distinct, i.e., in compositionally hot ICMEs.

To investigate the overall behavior of the average composition of ICMEs, Figure 3 plots $\mathrm{Mg} / \mathrm{O}, \mathrm{Si} / \mathrm{O}$, and $\mathrm{Ne} / \mathrm{O}$ as functions of $\mathrm{Fe} / \mathrm{O}$ for all ICMEs. This composition is derived from $2 \mathrm{hr}$ integrations of $A C E$ SWICS data with error bars that 

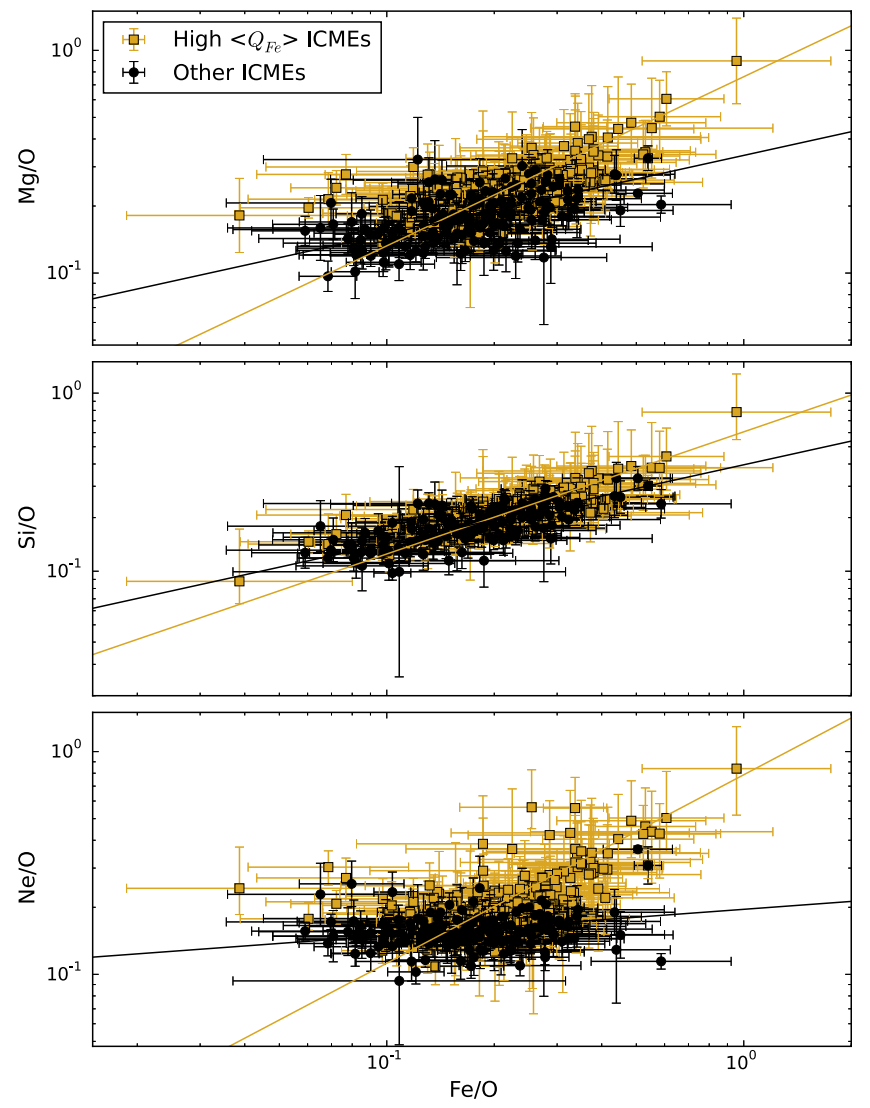

Figure 3. Composition of ICMEs for both compositionally hot ejecta (in orange) and ICMEs that are not compositionally hot. Error bars indicate the standard deviation of a given compositional quantity during the time of the ICME, as defined by Richardson and $\mathrm{Cane} . \mathrm{Mg} / \mathrm{O}$ and $\mathrm{Si} / \mathrm{O}$ are highly correlated with $\mathrm{Fe} / \mathrm{O}$, which may perhaps not be too surprising since they all have low FIP. Surprisingly, this correlation is also present in $\mathrm{Ne} / \mathrm{O}$ and $\mathrm{Fe} / \mathrm{O}$. For all elements, the slope for compositionally hot elements is steeper than that for the other ejecta.

indicate the standard deviation of each quantity within the ICME. We have used a total of 310 ICMEs on Richardson \& Cane's list from 1998 March, when the SWICS checkout period was complete and nominal operation began, until day 234 of 2011, after which SWICS was moved to a different operational mode because of a space weather-induced anomaly in the instrument's time-of-flight circuit. After that, a modified and reduced data set is available for SWICS, but was not included here.

In order to identify compositionally hot ICMEs, we used the methodology by Lepri et al. (2001), which classified that a given ICME be defined as "compositionally hot" if it contained a minimum of $6 \mathrm{hr}$ of plasma with $\left\langle Q_{\mathrm{Fe}}\right\rangle>12$. We note that this signature constitutes a compromise between the desire to reduce statistical outliers due to the limited counting statistics of our comparatively short-time accumulations and the desire to use time periods that are short as compared to the observed ICMEs. In contrast, Richardson \& Cane (2004) used a velocitydependent analysis of charge states. We note that some of the data used in their analysis have undergone substantial revision; a follow-up analysis should confirm whether the results persist in our new data, which are much more statistically robust. Because of the well-documented velocity changes of CMEs during their expansion through the corona into the heliosphere, the local velocity may or may not be a good measure of the
Table 1

Power-law Fits Shown in Figure 3 Computed Using Orthogonal Distance Regression

\begin{tabular}{lccccccc}
\hline \hline & \multicolumn{3}{c}{ Compositionally Hot } & & \multicolumn{2}{c}{ Not Compositionally Hot } \\
\cline { 2 - 4 } \cline { 6 - 8 } & $a$ & $b$ & $R^{2}$ & & $a$ & $b$ & $R^{2}$ \\
\hline $\mathrm{Mg} / \mathrm{O}$ & 0.77 & 0.77 & 0.58 & & 0.34 & 0.35 & 0.25 \\
$\mathrm{Si} / \mathrm{O}$ & 0.61 & 0.70 & 0.73 & & 0.40 & 0.44 & 0.57 \\
$\mathrm{Ne} / \mathrm{O}$ & 0.80 & 0.86 & 0.46 & & 0.20 & 0.12 & 0.04 \\
\hline
\end{tabular}

overall freeze-in speed, which is critically important for determining the ionic charge states (Gruesbeck et al. 2011). Therefore, we have avoided using such velocity-dependent signatures. Using our $\left\langle Q_{\mathrm{Fe}}\right\rangle$-based methodology, we identify $47.7 \%$ of the ICMEs in the list from Richardson \& Cane (2010) as compositionally hot.

The top panel of Figure 3 then displays $\mathrm{Mg} / \mathrm{O}$ as a function of $\mathrm{Fe} / \mathrm{O}$ for two classes of ICMEs: the compositionally hot ones plotted in orange, and the remaining ICMEs without the elevated $\left\langle Q_{\mathrm{Fe}}\right\rangle$ signature in black. Subsequent panels show $\mathrm{Si} /$ $\mathrm{O}$ and $\mathrm{Ne} / \mathrm{O}$ in the same format, all constituents that exhibit enhancements during ICMEs in Figure 2. The first result from this analysis is the breadth of the average compositional distribution for all compositional quantities extending over an order of magnitude from lowest to highest values. The fits plotted in Figure 3 are linear in log-space, i.e., of the form $\mathrm{Mg}$ / $\mathrm{O}=a(\mathrm{Fe} / \mathrm{O})^{b}$ for $\mathrm{Mg} / \mathrm{O}$, and analogous for the others, with results provided in Table 1 .

We first observe a positive correlation of $\mathrm{Mg} / \mathrm{O}$ and $\mathrm{Si} / \mathrm{O}$ with respect to $\mathrm{Fe} / \mathrm{O}$, pointing to the FIP effect being a robust physical process affecting metal-like ions in a similar fashion. The FIPs of $\mathrm{Mg}, \mathrm{Si}$, and Fe are, respectively, 7.65, 8.2, and $7.9 \mathrm{eV}$. It may therefore not be too surprising that the strongest correlations are found for $\mathrm{Si} / \mathrm{O}$ and $\mathrm{Fe} / \mathrm{O}$. The observed correlation is stronger for compositionally hot ICMEs than for the ICMEs that do not exhibit elevated ionic charge states.

The lowest panel of Figure 3 shows the composition of $\mathrm{Ne} /$ $\mathrm{O}$ as a function of $\mathrm{Fe} / \mathrm{O}$. Surprisingly, there is a strong positive correlation for $\mathrm{Ne} / \mathrm{O}$ for compositionally hot ICMEs. Such a correlation might not be expected since $\mathrm{Ne}$ is a high-FIP element with an FIP of $21.6 \mathrm{eV}$.

Table 1 also quantifies the degree of correlation for compositionally hot ICMEs and compares them with ICMEs that are not hot. Clearly, the elemental correlation is stronger in all cases for compositionally hot ICMEs and weak or absent for other ICMEs, especially when considering Ne.

Because of our stringent selection criteria for compositionally hot ICMEs, we should expect some "contamination" of moderately enhanced elemental abundances in ICMEs that are not categorized as compositionally hot. But even with these limitations, we conclude that compositionally hot ICMEs have a significantly different elemental composition than their cooler counterparts. To investigate such differences quantitatively and to compare them with the composition of other heliospheric plasmas, we compute the average composition of the two ICME populations and compare the values to those of both coronal hole-associated fast wind and slow wind associated with coronal streamers from Von Steiger \& Zurbuchen (2016).

The elemental composition relative to $\mathrm{O}$ is shown in Figure 4 and Table 2, which compare compositionally hot ICMEs and other ICMEs with the average composition of fast wind from coronal holes and slow wind associated with streamers. There 


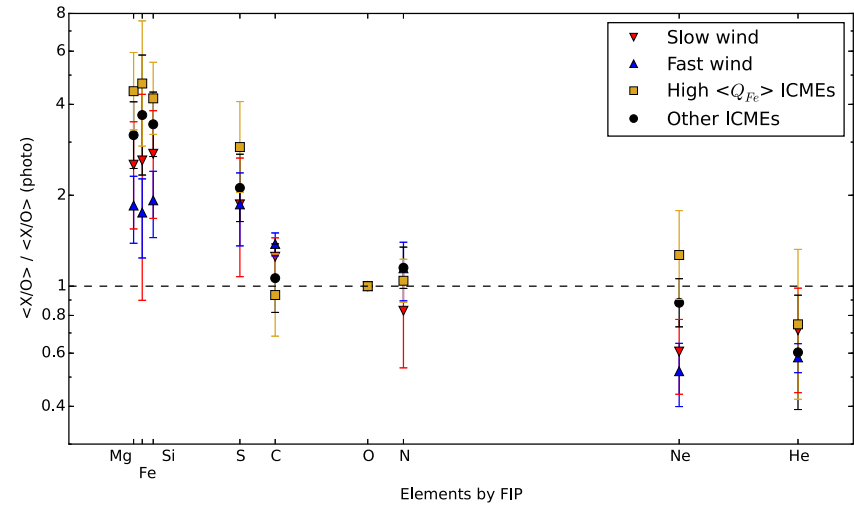

Figure 4. Elemental composition of compositionally hot ICMEs and other ICMEs as compared to the solar wind composition from fast, coronal holeassociated wind and from slow wind associated with streamers. Compositionally hot ICMEs have a higher FIP than both slow wind and other ICMEs. The $\mathrm{Ne} / \mathrm{O}$ composition of hot ICMEs exceeds that of both slow and fast wind.

are significant and systematically increasing FIP enhancement factors. The smallest FIP enhancement for $\mathrm{Mg}, \mathrm{Fe}$, and $\mathrm{Si}$ is seen for fast wind. The largest FIP factor is seen for compositionally hot ICMEs. The other samples are intermediate between these extremes.

The compositions of elements with intermediate FIP behave differently: S/O shows enhancements with an ordering identical to the metals, but with smaller enhancements and comparatively large error bars. The $\mathrm{C} / \mathrm{O}$ abundance shows a depletion as compared to average solar wind composition for both coronal hole-associated fast wind and streamer-associated slow wind. The depletions are relatively small, but significant in both compositionally hot ICMEs and in the remaining population of ICMEs that do not have the $\left\langle Q_{\mathrm{Fe}}\right\rangle$ signature. Within error bars, there is no significant difference between the two ICME populations for $\mathrm{C} / \mathrm{O}$. The remaining intermediate element, $\mathrm{N} / \mathrm{O}$, exhibits a small and probably insignificant enhancement over solar wind values. Note that the $\mathrm{N} / \mathrm{O}$ value used here from Von Steiger \& Zurbuchen (2016) differs from those of Von Steiger et al. (2000) due to improved data inversion techniques already discussed.

Finally, $\mathrm{Ne}$ and $\mathrm{He}$ are both noble gases with a rather peculiar behavior. The $\mathrm{Ne} / \mathrm{O}$ abundance ratio shows the enhancements already discussed in the context of Figure 3. Compositionally hot ICMEs exhibit an enhancement in $\mathrm{Ne} / \mathrm{O}$ that exceeds that of the solar wind by almost a factor of 2 . ICMEs without the $\left\langle Q_{\mathrm{Fe}}\right\rangle$ signature have a slightly elevated abundance as compared to streamer-associated slow wind, but it is significantly lower than the values of compositionally hot ICMEs.

Hot ICMEs have a slight enhancement in $\mathrm{He} / \mathrm{H}$, but the compositional anomalies of $\mathrm{He} / \mathrm{H}$ are much more intermittent than other compositional signatures, and the impact of these intermittent increases is modest when looked at in aggregate over entire ICMEs (Zurbuchen \& Richardson 2006).

In all cases, the composition of ICMEs that are not compositionally hot according to our analysis is intermediate between that of the compositionally hot ICMEs and that of slow, streamer-associated wind. This is indicative of actual intermediate states, but could also be an indication of crosscontamination of both compositions.

To determine a physical interpretation for these results, it is crucial to analyze the abundance of heavy ions relative to $\mathrm{H}$, as shown in Figure 5, by using $\mathrm{H} / \mathrm{O}$ values from Von Steiger \& Zurbuchen (2010). The compositional quantities in this figure are further normalized by the composition of polar coronal holes, which have been shown to be the most photosphere-like samples in the heliosphere (Von Steiger \& Zurbuchen 2016). To the best of our knowledge, the composition of polar coronal hole-associated wind exhibits only a small FIP effect andbesides $\mathrm{He} / \mathrm{H}$, which is depleted relative to that of the photosphere-fast solar wind is accelerated out to the low corona with no or minor fractionation effects.

Figure 5 shows that, to within the error bars, the abundance of low-FIP elements $(\mathrm{Fe} / \mathrm{H}, \mathrm{Si} / \mathrm{H}$, and $\mathrm{Mg} / \mathrm{H})$ of all solar wind samples shown is identical to that of coronal hole-associated wind. Similarly, $\mathrm{Ne} / \mathrm{H}$ for compositionally hot ICMEs is in very good agreement with that measured in high-latitude solar wind and somewhat reduced in both slow wind and ICMEs that are not compositionally hot.

Mid-FIP elements like $\mathrm{S}, \mathrm{C}, \mathrm{N}$, and $\mathrm{O}$ are reduced in all samples, with the most extreme example being $\mathrm{C} / \mathrm{H}$, in which compositionally hot ICMEs have less than $30 \%$ of the carbon content of fast wind from polar coronal holes. Similarly, the S/ $\mathrm{H}, \mathrm{O} / \mathrm{H}$, and $\mathrm{N} / \mathrm{H}$ values are depleted within both ICME samples, as compared to the slow wind. The FIP effect described in Figure 4 is, therefore, most likely due to depletion relative to $\mathrm{H}$, which can occur to different levels of depletion, and not to an enrichment of $\mathrm{Fe}, \mathrm{Si}$, and $\mathrm{Mg}$ relative to $\mathrm{H}$.

As previously reported, $\mathrm{He} / \mathrm{H}$ in slow wind is found to be slightly reduced in slow wind when compared to fast wind. Although we know that there are times of enhanced $\mathrm{He} / \mathrm{H}$ in ICMEs (Zurbuchen \& Richardson 2006), these do not compensate for an overall decrease in $\mathrm{He} / \mathrm{H}$ when integrating over all ICME samples.

\section{DISCUSSION}

Our abundance measurements of coronal mass ejections described above have critical implications for understanding several major aspects of coronal and heliospheric activity. The results yield new constraints on the nature of the FIP effect, on the source regions and the physical mechanisms for both ICMEs and the non-steady slow solar wind, and on ICMEinduced space weather effects, particularly SEPs.

With regard to the FIP bias, the most important conclusion is that the coronal abundance anomalies are due to the depletion of mid-FIP and high-FIP elements, not the enrichment of lowFIPs (Bochsler et al. 2000). This is clear from Figure 5, which shows that the abundance of low-FIP elements relative to $\mathrm{H}$ is approximately constant for all samples. The differences between high- and low-FIP elements are evident in S, C, N, and $\mathrm{O}$, which can be seen to be depleted to different magnitudes depending on the type of heliospheric region. Figure 5 also shows that $\mathrm{He}$ is similarly depleted relative to $\mathrm{H}$. The He depletion has been ascribed to be a consequence of insufficient Coulomb drag, which is responsible for the reduction in $\mathrm{He} / \mathrm{H}$ from $8.5 \%$ in the photosphere to $1 \%-6 \%$ in the heliosphere (Kasper et al. 2007). The effects of insufficient Coulomb drag are also observed in the isotopic composition of the solar wind (Heber et al. 2012), slightly decreasing the abundance of the heavier isotopes relative to the lighter ones.

The origin of the $\mathrm{S}, \mathrm{C}, \mathrm{N}$, and $\mathrm{O}$ depletions, on the other hand, is far from clear and is the subject of a great deal of ongoing research (e.g., Laming 2015 and references therein). 
Table 2

Average X/O Ratios in High $\left\langle Q_{\mathrm{Fe}}\right\rangle$ ICMEs and Other Solar Wind Plasmas Relative to the Photosphere ${ }^{\mathrm{a}}$

\begin{tabular}{lccccccc}
\hline \hline & $\mathrm{He} / \mathrm{O}$ & $\mathrm{C} / \mathrm{O}$ & $\mathrm{N} / \mathrm{O}$ & $\mathrm{Ne} / \mathrm{O}$ & $\mathrm{Mg} / \mathrm{O}$ & $\mathrm{Si} / \mathrm{O}$ & $\mathrm{S} / \mathrm{O}$ \\
\hline Slow solar wind $^{\mathrm{b}}$ & 0.715 & 1.252 & 0.830 & 0.607 & 2.525 & 2.744 & 1.866 \\
Errors $^{\text {Fast solar wind }}{ }^{\mathrm{b}}$ & 0.271 & 0.194 & 0.293 & 0.169 & 0.978 & 1.067 & 0.791 \\
Errors & 0.581 & 1.380 & 1.146 & 0.523 & 1.849 & 1.925 & 1.866 \\
High $\left\langle Q_{\mathrm{Fe}}\right\rangle$ ICMEs & 0.064 & 0.121 & 0.252 & 0.124 & 0.462 & 0.477 & 0.506 \\
Error range min. & 0.748 & 0.935 & 1.042 & 1.268 & 4.423 & 4.188 & 2.889 \\
Error range max. & 0.325 & 0.252 & 0.158 & 0.364 & 1.131 & 1.007 & 0.847 \\
Other ICMEs & 0.576 & 0.344 & 0.186 & 0.511 & 1.519 & 1.325 & 1.197 \\
Error range min. & 0.604 & 1.063 & 1.151 & 0.881 & 3.163 & 3.436 & 2.116 \\
Error range max. & 0.213 & 0.244 & 0.168 & 0.148 & 0.710 & 0.749 & 0.479 \\
\hline
\end{tabular}

Notes.

${ }^{a}$ All values are normalized relative to the photospheric values of Grevesse \& Sauval (1998).

${ }^{\mathrm{b}}$ Average values for fast and slow wind from Von Steiger \& Zurbuchen (2016).

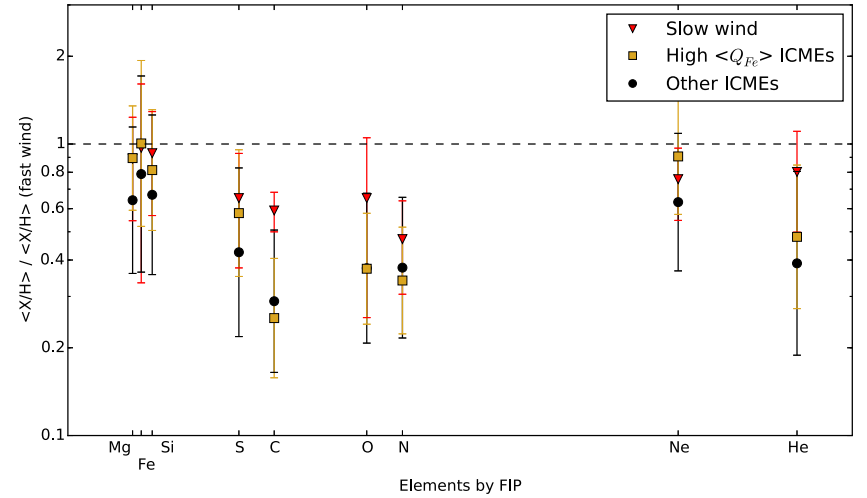

Figure 5. Elemental composition of slow wind, high $\left\langle Q_{\mathrm{Fe}}\right\rangle$ ICMEs, and other ICMEs as compared to that of solar wind from high-latitude coronal holes, which is the most photosphere-like sample available in the heliosphere. Compositionally hot ICMEs are depleted in $\mathrm{S}, \mathrm{C}, \mathrm{O}$, and $\mathrm{He}$, but, to within error bars, are identical to that of polar coronal holes for $\mathrm{Mg}, \mathrm{Si}, \mathrm{Fe}$, and $\mathrm{Ne}$.

Our in situ composition results appear to be in good agreement with remote-sensing spectroscopic data on delineating the main properties of the FIP effect mechanism. The essential conclusion from the spectroscopic observations is that the FIP bias in the corona has a distinct time dependence (e.g., Sheeley 1995; Widing \& Feldman 2001). When flux first emerges into the corona, as either a small bipole of the magnetic carpet or a larger bipole of an active region, the plasma exhibits photospheric abundances, but over a timescale of $10^{5}-10^{6} \mathrm{~s}$ the loop plasma acquires the coronal FIP bias (Widing \& Feldman 2001). An important point is that this timescale is long compared to the coronal ejection time of either CME or streamer-top plasma, $\sim 10^{3} \mathrm{~s}$; consequently, the in situ measurements are not likely to be due to any ejection dynamic or heating, but must be due to the long-term preejection evolution in the corona. This point is further confirmed by Hinode spectroscopic observations showing that flare plasma, which is evaporated from the chromosphere on the rapid timescale of the ejection itself, exhibits photospheric abundances (Warren 2014). The plasma ejected in the CME, however, is material that existed in the active region corona, most often for several days, prior to eruption. The elevated charge states observed are thus a reflection of these initial conditions (i.e., higher temperatures, higher densities, etc.) but also a reflection of the heating and expansion process of the ICME (see, e.g., Gruesbeck et al. 2011).

Widing \& Feldman (2001) studied the evolution of coronal FIP bias in detail and concluded that it increases approximately linearly with time, but as pointed out by Baker et al. (2015), this linear increase is generally counteracted by the mixing of plasmas due to reconnection of active region loops with newly emerged bipoles or with neighboring flux systems, especially if this neighboring flux is open. Since flux emergence is ubiquitous throughout the Sun, we expect that the strongest FIP bias is to be found in the cores of active regions where the strong magnetic field undergoes little evolution, except for the occasional eruption, for timescales of days.

The results of Figure 4 directly confirm this dependence of the FIP bias on time. We note that fast wind plasma, which typically spends the least amount of time in the corona, $<1$ day, shows the smallest FIP bias, while the hot ICME plasma, which is expected to originate from active region cores, shows the largest bias. The slow wind and the other ICMEs show FIP effects intermediate between these two, as would be expected if these originate in closed field regions outside active regions where there is mixing of coronal plasma with photospheric due to the ubiquitous emergence of flux on supergranular timescales, 1-2 days. This is consistent with the latitude dependence of observations of ICMEs with elevated charge states, as reported by Lepri \& Zurbuchen (2004). Our results strongly support the scenario that the FIP effect is due to some mechanism that constantly depletes high-FIP elements from coronal loops. This mechanism begins to operate soon after the loop flux has emerged and continues until the loop is destroyed by reconnection with other flux. The magnitudes of the observed FIP biases, therefore, appear to be due to the lifetimes of coronal flux rather than to some intrinsic saturation limit in the FIP mechanism itself.

We conclude from the discussion above that the in situ composition measurements provide a unique probe of not only the location in the corona of the heliospheric plasma's source, but also the dynamics of this source region. We can use this result to gain insight into the nature of the slow wind. The data indicate that active region loops are not a significant source of the background slow solar wind, but ICME eruptions do explosively open the overlying magnetic field and carry active region plasma out into the heliosphere (Antiochos et al. 1999). This is a strong constraint on models for the slow, streamerassociated wind. Recent models by Fisk \& Zurbuchen (2006) 
and Antiochos et al. (2011) suggest the importance of interchange reconnection for releasing plasma from the magnetically closed corona into the heliosphere. However, the exact locations and detailed topology of the release and acceleration processes remain under debate. For example, Antiochos predicts that there is a strong preference for such reconnection to occur at the open-closed boundary in the corona, which maps out to the heliospheric current sheet and to more generalized singular layers in the heliosphere, referred to as S-web (Antiochos et al. 2011). In the interchange models of Fisk and co-workers, on the other hand, the reconnection is postulated to occur throughout the closed field corona.

Our observations are in qualitative agreement with the S-web model: active region loops are not sources of the heliosphere under quasi-steady conditions, but are only ejected within CMEs. This conclusion is consistent with the UV/EUV spectroscopic observations by Feldman \& Widing (2003), who report a large FIP bias as in our data for mature active regions, lower biases for quiet regions, and essentially no FIP effect in coronal holes. Interestingly enough, Feldman \& Widing further indicate that there are cases where $\mathrm{Ne}$ is enhanced over the photosphere, consistent with our interpretation. In contrast, large-scale loops near coronal boundaries, as identified by an elemental composition that reflects gravitational settling, routinely and repeatedly become sources of solar wind plasma (Weberg et al. 2012), as predicted by the S-web theory. Our results also imply that the slow wind must be released from loops whose lifetimes are of intermediate timescale, long enough to build up a significant FIP effect but not as long as the several-day lifetime of active region loops. Again, this is consistent with a picture in which a closed field near the open-closed boundary undergoes a cycle of opening and closing on supergranular timescales, 1-2 days, as in the S-web.

Earlier we pointed out that ICMEs and the particles they accelerate are a critical source of space radiation in the form of SEPs. The standard model for particle acceleration in large SEP events is shock acceleration (e.g., Jokipii 1982) in which a CME-driven shock propagates ahead of the CME into the ambient solar wind and accelerates particles from suprathermal to high energies. In this case the shock acceleration process is presumed to be responsible for the significant mass-per-charge fractionation observed in SEP events enriched in Fe and other heavy elements (Cohen et al. 1999; Tylka et al. 2005; Reames 2015).

Alternative theories, however, have suggested the importance of local acceleration, such as through stochastic processes that lift ions from the thermal background (Fisk et al. 2010). Gruesbeck et al. (2015) recently showed experimental evidence for local acceleration of ions up to $200 \mathrm{keV} / \mathrm{e}$ in conjunction with ICMEs near $1 \mathrm{AU}$ : compositional signatures of the suprathermal particles were much more similar to those of plasma within the ICME than to the composition of the solar wind in which the ICME dynamically interacts. Note also that in the inner heliosphere, suprathermal populations are an important source of seed particles for shock acceleration processes. For example, remnant materials from impulsive ${ }^{3} \mathrm{He}$ rich events have been shown to make important contributions to the acceleration of many CME-driven events (Mason et al. 1999; Reames 2014), which may also explain why the mean charge state of $\mathrm{Fe}$ in these events often ranges from +15 to +20 (Mazur et al. 1998; Moebius et al. 1998; Tylka et al. 2005), indicative of high-temperature material. High $\left\langle Q_{\mathrm{Fe}}\right\rangle$ ICME material has similar properties (see Table 2).

The relevant acceleration mechanisms can occur in a number of scenarios for the near-Sun evolution of ICMEs. For example, Roelof (2015) has presented a model in which SEPs can undergo compressive acceleration within CMEs in an extended region behind the shock. Also, Li et al. (2011) have discussed a twin-CME scenario in which reconnection processes could enable ICME material from a first CME to become accessible for acceleration by the shock driven by a second CME (see Kahler \& Vourlidas 2014 for a review). See also Zank et al. (2015) for a discussion of a modified diffusive shock acceleration process that includes the impact of magnetic reconnection and resulting plasmoids, which may also be of importance in this context.

Our results have important implications for theories that seek to explain the acceleration of SEP particles near ICMEs, because the composition of the plasma within which the acceleration process occurs is clearly an important ingredient. According to our analysis, many ICMEs are Fe-rich and also have enhanced $\mathrm{Ne} / \mathrm{O}$ ratios, both found in SEP events associated with CME-driven shocks (Mewaldt et al. 2007; Reames 2015). It is therefore worth investigating the possible contributions of ICME material to Fe-rich SEPs, in addition to remnant flare material and other sources. If plasma in ICMEs is an important contributor to the source of SEPs accelerated in their vicinity, one might also expect enrichments for $\mathrm{Ne}, \mathrm{Mg}$, $\mathrm{Si}$, and a possible depletion of $\mathrm{He}$. It should be possible to test these conjectures with future studies of ICME and SEP composition, such as those expected from the upcoming Solar Orbiter and Solar Probe Plus missions.

We acknowledge the work of the ACE SWICS team and particularly Paul Shearer for their dedication to producing highquality compositional data. T.H.Z. acknowledges the hospitality of the staff of the International Space Science Institute where much of this work was performed. This work was supported, in part, by NASA grants NNX13AH66G, NNH13ZDA001N, and NNX11A075G, and the Catholic University of America contract 362496-Sub1.

\section{REFERENCES}

Antiochos, S. K., DeVore, C. R., Karpen, J. T., \& Mikić, Z. 2007, ApJ, 671, 936

Antiochos, S. K., DeVore, C. R., \& Klimchuk, J. A. 1999, ApJ, 510, 485

Antiochos, S. K., Mikić, Z., Titov, V. S., Lionello, R., \& Linker, J. A. 2011, ApJ, 731, 112

Asplund, M. 2005, ARA\&A, 43, 481

Baker, D., Brooks, D. H., Démoulin, P., et al. 2015, ApJ, 802, 104

Bochsler, P., Ipavich, F. M., Paquette, J. A., Weygland, J. M., \& Wurz, P. 2000, JGR, 105, 12659

Borrini, G., Gosling, J. T., Bame, S. J., \& Feldman, W. C. 1982, JGR, 87, 7370 Cohen, C. M. S., Mewaldt, R. A., Leske, R. A., et al. 1999, GeoRL, 26, 2697 Feldman, U., \& Widing, K. G. 2003, SSRv, 107, 665

Fisk, L. A., Gloeckler, G., \& Schwadron, N. A. 2010, ApJ, 720, 533

Fisk, L. A., \& Zurbuchen, T. H. 2006, JGR, 111, A09115

Geiss, J., Gloeckler, G., von Steiger, R., et al. 1995, Sci, 268, 1033

Gilbert, J. A., Gershman, D. J., Gloeckler, G., et al. 2014, RScI, 85, 091301

Gloeckler, G., Cain, J., Ipavich, F. M., et al. 1998, SSRv, 86, 497

Grevesse, N., \& Sauval, A. J. 1998, SSRv, 85, 161

Gruesbeck, J. R., Lepri, S. T., \& Zurbuchen, T. H. 2012, ApJ, 760, 141

Gruesbeck, J. R., Lepri, S. T., Zurbuchen, T. H., \& Antiochos, S. K. 2011, ApJ, 730,104

Gruesbeck, J. R., Lepri, S. T., Zurbuchen, T. H., \& Christian, E. R. 2015, ApJ, 799, 57

Heber, V. S., Baur, H., Bochsler, P., et al. 2012, ApJ, 759, 121 
Henke, T., Woch, J., Schwenn, R., et al. 2001, JGR, 106, 10597

Hirshberg, J., Bame, S. J., \& Robbins, D. E. 1972, SoPh, 23, 467

Ipavich, F. M., Galvin, A. B., Gloeckler, G., Hovestadt, D., \& Klecker, B. 1986, JGR, 91, 4133

Jokipii, J. R. 1982, ApJ, 255, 716

Kahler, S. W., \& Vourlidas, A. 2014, ApJ, 784, 47

Kasper, J. C., Stevens, M. L., Lazarus, A. J., Steinberg, J. T., \& Ogilvie, K. W. 2007, ApJ, 660, 901

Laming, J. M. 2015, LRSP, 12, 2

Lepri, S. T., \& Zurbuchen, T. H. 2004, JGR, 109, A01112

Lepri, S. T., Zurbuchen, T. H., Fisk, L. A., et al. 2001, JGR, 107, 29231

Li, G., Moore, R., Mewaldt, R. A., et al. 2011, SSRv, 171, 141

Mason, G. M., Mazur, J. E., Dwyer, J. W., et al. 1999, ApJL, 525, L133

Mazur, J. E., Mason, G. M., Looper, M. D., et al. 1998, GeoRL, 26, 173

Mewaldt, R. A., Cohen, C. M. S., Mason, G. M., et al. 2007, SSRv, 130, 207

Moebius, E., Popecki, M., Klecker, B., et al. 1998, GeoRL, 26, 145

Reames, D. 1994, AdSpR, 14, 177

Reames, D. V. 2014, SoPh, 289, 977

Reames, D. V. 2015, SSRv, 194, 303
Reinard, A. 2005, ApJ, 620, 501

Richardson, I. G., \& Cane, H. V. 2004, JGR, 109, A09104

Richardson, I. G., \& Cane, H. V. 2010, SoPh, 264, 189

Roelof, E. C. 2015, JPhCS, 642, 012023

Shearer, P., von Steiger, R., Raines, J. M., et al. 2014, ApJ, 789, 60

Sheeley, N. R. 1995, ApJ, 440, 884

Tylka, A. J., Cohen, C. M. S., Dietrich, W. F., et al. 2005, ApJ, 625, 474

Von Steiger, R., Schwadron, N. A., Fisk, L. A., et al. 2000, JGR, 105, 27217

Von Steiger, R., \& Zurbuchen, T. H. 2010, GeoRL, 37, L22101

Von Steiger, R., \& Zurbuchen, T. H. 2016, ApJ, 816, 13

Warren, H. P. 2014, ApJL, 786L, 2W

Weberg, M. J., Zurbuchen, T. H., \& Lepri, S. T. 2012, ApJ, 760, 30

Widing, K. G., \& Feldman, U. 2001, ApJ, 555, 426

Zank, G. P., Hunana, P., Mostafavi, P., et al. 2015, ApJ, 814, 137

Zhao, L., Landi, E., Lepri, S. T., Kocher, M., \& Fisk, L. A. 2016, ApJS, submitted

Zurbuchen, T. H. 2007, A\&A, 45, 297

Zurbuchen, T. H., Gloeckler, G., Ipavich, F., et al. 2004, GeoRL, 31, L11805

Zurbuchen, T. H., \& Richardson, I. G. 2006, SSRv, 123, 31 